\title{
THE LIS STUDY (LYUBERTSY STUDY ON MORTALITY RATE IN PATIENTS AFTER ACUTE MYOCARDIAL INFARCTION). EVALUATION OF DRUG THERAPY. PART 2. INFLUENCE OF PREVIOUS DRUG TREATMENT ON LONG-TERM LIFE PROGNOSIS
}

\author{
S.Yu. Martsevich1,2, M.L.Gynzburg33, N.P. Kutishenko1,2, A.D. Deev1, V.P. Smirnov³, L.U. Drozdova1, \\ E.V. Daniels3 ${ }^{3}$ A.V. Fokina 3 \\ 1State Research Center for Preventive Medicine. Petroverigsky per. 10, Moscow, 101990, Russia \\ 2I.M. Sechenov First Moscow State Medial University. Trubetskaya ul. 8-2, Moscow, 119991, Russia \\ 3Lyubertsy Regional Hospital №2. Oktyabr'skiy prospect 338, Lyubertsy, 140006, Russia
}

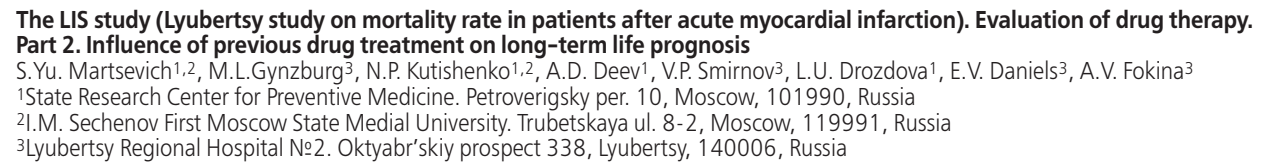

Aim. To evaluate drug therapy received by patients who had survived acute myocardial infarction (AMI) in the framework of the AMI register (the "LIS" study) and estimate this therapy influence on long-term outcomes of the disease.

Material and methods. The total of 961 patients of 1133 enrolled in the "LIS" study, were discharged from hospital. 191 patients had died during follow-up. 632 patients (who had survived and consented to visit out-patient clinic) underwent repeated examination (median of follow-up 1.6 [1.0; 2.4] years). Data about treatment before and during AMI were received from patient's charts; data about treatment after AMI were obtained from out-patient medical records.

Results. Before reference AMI only a small number of the patients received the main drug groups (antiplatelet agents, $\beta$-blockers, ACE inhibitors, statins), at that ACE inhibitors were prescribed more often than the others. Use of $\beta$-blockers and ACE inhibitors before reference AMI significantly improved long-term life prognosis [relative risk (RR) 0.70 and 0.66 , respectively]. Rate of the main drug groups prescribed in hospital was rather high with the exception of thrombolytics (less than 10\%). Thrombolytics, $\beta$-blockers and antiplatelet agents prescribed in hospital significantly improved long-term life prognosis of patients (RR $0.42,0.65$ and 0.58 respectively). At the second visit (according to data of out-patient medical records) rate of antiplatelet agents, ACE inhibitors, $\beta$-blockers and statins prescription exceeded $60 \%$.

Conclusion. Very low prevalence of adequate drug therapy preceding AMI determines high mortality rate among survived acute stage of myocardial infarction patients in long-term period. Key words: acute myocardial infarction, register, long-term life prognosis, evaluation of drug treatment.

Rational Pharmacother. Card. 2012;8(5):738-745

Исследование ЛИС (Люберецкое исследование смертности больных, перенесших острый инфаркт миокарда). Оценка лекарственной терапии.

Часть 2. Влияние предшествующей лекарственной терапии на отдаленный прогноз жизни больных

С.Ю. Марцевич1,2, М.Л. Гинзбург ${ }^{3}$, Н.П. Кутишенко 1,2, А.Д. Деев¹, В.П. Смирнов³, Л.Ю. Дроздова1, Е.В. Даниэльс ${ }^{3}$, А.В. Фокиназ

${ }_{1}^{1}$ Государственный научно-исследовательский центр профилактической медицины. 101990, Москва, Петроверигский пер., 10

2 Первый Московский медицинский университет им. И.М. Сеченова. 119991, Москва, ул. Трубецкая, 8, стр. 2

3 Люберецкая районная больница №2. 140006, Люберцы, Октябрьский проспект, 338

Цель. В рамках регистра острого инфаркта миокарда (ОИМ; исследование ЛИС) оценить лекарственную терапию, которую получали больные, выжившие после ОИМ, и оценить ее влияние на отдаленные исходы заболевания

Материал и методы. Из 1133 больных, включенных в исследование лИС, 961 был выписан из стационара. За время наблюдения умер 191 человек. 632 человека (выжившие и согласившиеся на визит в поликлинику) были осмотрены повторно [медиана наблюдения 1,6 (1,0; 2,4) года]. Данные о терапии, проводимой до и во время ОИМ, получены из историй болезни; данные о терапии в отдаленные сроки получены из амбулаторных карт.

Результаты. До развития референсного ОИМ основные группы лекарственных препаратов (антиагреганты, бета-адреноблокаторы (БАБ), ингибиторы ангиотензинпревращающего фермента (ИАПФ), статины] принимала лишь небольшая часть больных, причем чаще других назначались ИАПФ. Прием БАБ и ИАПФ перед референсным ОИМ значимо улучшал отдаленный прогноз жизни [относительный риск (OP) 0,70 и 0,66, соответственно]. В стационаре частота назначения основных групп лекарственных препаратов оказалась достаточно высокой, за исключением тромболитиков (менее 10\%). Тромболитики, БАБ и антиагреганты, назначенные в стационаре, достоверно улучшали отдаленный прогноз жизни больных (ОР 0,42, 0,65 и 0,58, соответственно). При повторном визите частота назначения (по данным амбулаторных карт) антиагрегантов, ИАПФ, БАБ, статинов превышала $60 \%$.

Заключение. Крайне невысокий охват адекватной лекарственной терапией перед развившимся ОИМ в значительной степени определяет высокие показатели смертности в отдаленном периоде у выживших после острой стадии инфаркта миокарда.

Ключевые слова: острый инфаркт миокарда, регистр, отделенный прогноз жизни, оценка лекарственной терапии.

РФК 2012;8(5):738-745

*Corresponding author (Автор, ответственный за переписку): smartsevich@gnicpm.ru

\section{Authors' information:}

Sergey Yu. Martsevich - PhD, MD, Head of Department of Preventive Pharmacotherapy, State Research Center for Preventive Medicine; Professor of Chair of Evidence-based Medicine, I.M. Setchenov First Moscow State Medical University

Moisei L. Ginzburg - PhD, MD, Head of Cardiology Department, Lyubertsy Regional Hospital №2

Natalia P. Kutishenko - PhD, MD, Head of Laboratory of Preventive Pharmacotherapy, Department of Preventive Pharmacotherapy, State Research Center for Preventive Medicine; Professor of Chair of Evidence-based Medicine, I.M. Setchenov First Moscow State Medical University

Alexander D. Deev - PhD, Head of Laboratory of Biostatistics, Department of Preventive Pharmacotherapy, State Research Center for Preventive Medicine

Vladimir P. Smirnov - PhD, MD, Head of Lyubertsy Regional Hospital №2 Lyubov Yu. Drozdova - PhD, MD, Researcher of Department of Preventive Pharmacotherapy, State Research Center for Preventive Medicine

Elena V. Daniels - MD, Doctor of Cardiology Department, Lyubertsy Regional Hospital №2

Anna V. Fokina - MD, Doctor of the same Department
Сведения об авторах:

Марцевич Сергей Юрьевич - д.м.н., проф., руководитель отдела профилактической фармакотерапии ГНИЦ ПМ, профессор кафедры доказательной медицины Первого МГМУ им. И.М.Сеченова

Гинзбург Моисей Львович - К.м.Н., зав. кардиологическим отделением Люберецкой районной больницы №2

Кутишенко Наталья Петровна - Д.м.Н., зав. лабораторией отдела профилактической фармакотерапии ГНИЦ ПМ; профессор кафедры доказательной медицины Первого МГМУ им. И.М.Сеченова

Деев Александр Дмитриевич - к.ф-м.н., зав. лабораторией биостатистики отдела профилактической фармакотерапии ГНИЦ ПМ Смирнов Владимир Павлович - К.м.Н., главный врач Люберецкой районной больницы №2

Дроздова Любовь Юрьевна - К.М.Н., Н.с. отдела профилактической фармакотерапии ГНИЦ ПМ

Даниэльс Елена Викторовна - врач кардиологического отделения

Люберецкой районной больницы №2

Фокина Анна Валериановна - врач того же отделения 
Significant progress has been achieved in ischemic heart disease (IHD) treatment in last decades. A number of controlled clinical trials (CCT) have demonstrated that some drugs such as antiplatelet agents, $\beta$-blockers, angiotensin-converting enzyme (ACE) inhibitors, statins can significantly reduce probability of the IHD complications and improve prognosis for life in this disease [1 -6]. Decrease in IHD mortality rate, registered in a number of countries, is to a considerable degree explained by widespread implementation of these medications in clinical practice [7].

It is well known that despite evident advantages of these drugs not all the patients they are indicated in really receive these preparations [8-12]. The most reliable way to estimate real clinical practice is analyzing of diseases registers that also allow to evaluate correspondence of prescribed treatment to modern clinical guidelines [13-15]. With help of registers one can often evaluate efficacy of one or another drug owing to natural formation of groups of patients who do or do not receive any specific preparation $[16,17]$.

The aim of this work, carried out in the framework of the "LIS" study, was to evaluate medical treatment in patients who had survived acute myocardial infarction (AMI). We also tried to estimate how prescribed therapy influences the long-term outcomes of the disease.

\section{Materials and methods}

The design and protocol of the "LIS" study have been described earlier $[18,19]$. All the cases of confirmed AMI in hospitals of Lyubertsy district of Moscow Region from 01.01.2005 to 31.12.2007 were enrolled into the "LIS" study. The total of 1133 patients with confirmed AMI (it was called the reference AMI) were recruited. 172 patients died in hospital and 961 were discharged - this particular subgroup of patients became a subject of investigation in prospective part of the "LIS" study, which had started in 2008. We attempted to come in contact with each patient of those 961 , first of all to determine their life status. The primary endpoint of the "LIS" study was all-cause mortality in the patients. If an attempt to contact with a patient (or his relatives) was successful he (she) was invited for the repeated examination. At that apart from standard examination a number of data were ascertained (information of risk factors, received drugs and so on). In case of death of a patient we tried to specify its cause (using medical documents, including autopsy results) and circumstances (at contact with relatives).

All the data concerning history of the disease, its diagnostics and treatment prescribed during reference AMI were received from patient's charts. All the
В лечении ишемической болезни сердца (ИБС) за последние десятилетия достигнуты значительные успехи. В ряде контролируемых клинических исследований (ККИ) доказано, что ряд лекарственных препаратов - антиагрегантов, бета-адреноблокаторов (БАБ), ингибиторов ангиотензинпревращающего фермента (ИАПФ), статинов - способны существенно снизить вероятность осложнений ИБС и улучшить прогноз жизни при данном заболевании [1-6]. Зафиксированное в ряде стран снижение смертности от ИБС на популяционном уровне в значительной степени объясняется широким внедрением этих препаратов в повседневную клиническую практику [7].

Хорошо известно, что, несмотря на очевидную пользу от применения этих препаратов, их по разным причинам получают далеко не все больные, имеющие прямые показания к их назначению [8-12]. Наиболее надежным способом оценить реальную клиническую практику являются регистры заболеваний, позволяющие, в том числе, выявить, насколько получаемая больными терапия соответствует современным клиническим рекомендациям [13-15]. В рамках регистров нередко удается оценить эффективность тех или иных препаратов, как правило, за счет того, что естественным путем формируются группы, получающие и не получающие конкретный лекарственный препарат $[16,17]$.

Целью данной работы, выполнявшейся в рамках исследования ЛИС, являлась оценка лекарственной терапии, которую получали больные, выжившие после острого инфаркта миокарда (ОИМ). Была предпринята также попытка оценить, как принимаемая терапия может повлиять на отдаленные исходы заболевания.

\section{Материал и методы}

Дизайн и протокол исследования ЛИС подробно описаны ранее $[18,19]$. Исследование ЛИС включило все случаи диагноза ОИМ, подтвержденного в стационарах (Люберецкий район Московской области) за период с 1 января 2005 г. по 31 декабря 2007 г. Всего было включено 1133 больных, у которых при госпитализации подтвердился диагноз ОИМ (он был назван референсным ОИМ). В стационаре умерли 172 человека, 961 человек были выписаны из стационара - именно эта подгруппа больных явилась предметом изучения в проспективной части исследования ЛИС, начавшейся в 2008 г. Была предпринята попытка установить контакт с каждым из 961 больного, в первую очередь, для определения жизненного статуса. Первичной конечной точкой исследования лИС была общая смертность больных. Если с пациентом (или с его родственниками) удавалось установить контакт, то его приглашали для повторного обследования, во время которого, кроме стандартного обследования, уточняли ряд данных (в частности, информацию о факторах риска, получаемом на момент визита лечении и др.). В случае смерти пациента пытались установить ее причину (по медицинским документам, в том числе, по результатам вскрытия) и обстоятельства (при контакте с родственниками). 
data of therapy administrated at late terms after the reference AMI were received from out-patient medical records and filled in a chart of follow-up at the second visit.

Statistical analysis was carried out using the SAS (Statistical Analysis System). For survival rate modeling and evaluation of drugs prognostic value we used Cox proportional hazard regression model realized in the SAS PROC PHREG procedure. A macroprocedure was used that allowed to test for statistical significance many continuous, categorical and categorized indices with taking into account influence of set of invariably significant indices (such as gender and age) that were included in the model forcibly. Results were presented as relative risk (RR). Significance of testable variables was evaluated using Wald $\chi^{2}$ test.

\section{Results}

Life status of 850 persons of 961 discharged patients was determined. We failed to ascertain it in the rest of 111 persons $(11.6 \%$ of all the patients enrolled into this part of the study). The total of 191 patients of above mentioned 850 had died (19.9\% of all the discharged from hospital); other 659 were invited for the repeated examination. $27 \mathrm{pa}$ tients could not come due to different reasons. The median of follow-up for these patients was 1.6 (1.0; 2.4) years.

Table 1 shows main clinical and anamnestic characteristics of 961 patients who had survived reference AMI. Influence of these factors on life prognosis was in detail discussed in previous publications [20]. However, it should be noted that patients survived the reference $\mathrm{Ml}$ apparently had high risk of its advent: majority of them had previously suffered from the IHD (69.2\%), this AMI was recurrent in $20.8 \%$ of the patients and many patients had almost the whole set of risk factors.

Data on medications received by the enrolled patients before the reference AMI are shown in the Table 2. As it follows from Table 2, firstly, only a small part of the patients received the main groups of drugs (antiplatelet agents, $\beta$-blockers, ACE inhibitprs, statins), at that ACE inhibitors were prescribed more often than the others. Secondly, the data distinctly testify that some drugs received before the AMI had benefited life prognosis in long-term period. It was primarily regarded to $\beta$-blockers and ACE inhibitors (RR 0.70 and 0.66 , respectively).

Table 3 presents data on medical treatment in the survived AMI patients in acute stage of the disease. Rate of the main drug groups prescription, which are proved to influence life prognosis (antiplatelet agents, $\beta$-blockers, ACE inhibitors, statins) was
Все данные, касающиеся анамнеза, диагностики заболевания, назначенного лечения во время референсного ОИМ были получены из историй болезни. Все данные о лечении, получаемом в отдаленные сроки после референсного ОИМ, получали при повторном визите, эти данные вносились в карту наблюдения лечащими врачами из амбулаторных карт больных.

Статистический анализ проводился в системе SAS (Statistical Analysis System). Для моделирования выживаемости и определения прогностического влияния лекарственных препаратов использовалась регрессионная модель пропорционального риска (Кокса), реализованная в процедуре SAS PROC PHREG. Была использована макропроцедура, позволяющая тестировать на значимость множество непрерывных, категориальных или категоризованных показателей при учете влияния заведомо значимых показателей (пол и возраст), включаемых в модель принудительно. Результаты представлялись в виде относительного риска (ОР). Значимость тестируемых переменных определялась с помощью критерия $\chi^{2}$ Вальда.

\section{Результаты}

Из 961 пациента, выписанных домой из стационара, жизненный статус был установлен у 850 человек, у 111 человека жизненный статус остался неизвестным, что составило 11,6\% от всех больных, включенных в эту часть исследования. Из указанных выше 850 человек умерли 191 (19,9\% от общего числа выписанных из стационара), остальные 659 были приглашены для повторного осмотра, 27 пациентов по различным причинам не смогли явиться на визит. Медиана наблюдения для этих пациентов составила 1,6 (1,0; 2,4) лет.

В табл. 1 представлены основные клинико-анамнестические характеристики 961 больного, перенесшего референсный ОИМ. Влияние данных факторов на прогноз жизни больных было подробно рассмотрено в других публикациях [20]. Отметим лишь то, что выжившие после референсного ОИМ пациенты явно имели высокий риск его развития: большинство из них до этого страдали ИБС $(69,2 \%)$, а у 20,8\% пациентов данный ОИМ был повторным, у многих больных имелся почти полный набор факторов риска.

В табл. 2 приведены данные о лекарственной терапии, которую принимали включенные в эту часть исследования больные перед референсным ОИМ. Из таблицы следует, вопервых, что основные группы лекарственных препаратов (антиагреганты, БАБ, ИАПФ, статины) принимала лишь небольшая часть больных, причем чаще других назначались ИАПФ. Во-вторых, отчетливо видно, что некоторые группы препаратов, принимаемые до ОИМ, оказали положительное влияние на прогноз жизни и в отдаленные сроки. В первую очередь, это касалось БАБ и ИАПФ (ОР 0,70 и 0,66, соответственно).

В табл. 3 представлены данные о лекарственной терапии выживших пациентов после ОИМ в острой стадии болез- 
Table 1. Characteristics of 961 AMI patients discharged from hospital

Таблица 1. Характеристика 961 больного, выписанных из стационара после ОИМ

\begin{tabular}{|c|c|}
\hline Index / Признак & Value / Значение \\
\hline Men/Мужчины, n (\%) & $540(56.2)$ \\
\hline Women/Женщины, n (\%) & $421(43.8)$ \\
\hline $\begin{array}{l}\text { Mean age, among them/Средний возраст, в т.ч. } \\
\text { men/мужчины } \\
\text { women/женщины }\end{array}$ & $\begin{array}{l}63.9 \pm 0.4 \\
59.0 \pm 0.5 \\
70.2 \pm 0.5\end{array}$ \\
\hline Arterial hypertension/Артериальная гипертония, n (\%) & $731(76.1)$ \\
\hline Hypercholesterolemia/Гиперхолестеринемия, n (\%) & $347(36.1)$ \\
\hline Smoking/Курение, n (\%) & $298(31.0)$ \\
\hline Sedentary lifestyle/Малоподвижный образ жизни, n (\%) & $596(62.0)$ \\
\hline Obesity/Ожирение, n (\%) & $386(40.2)$ \\
\hline Diabetes mellitus/Сахарный диабет, n (\%) & $154(16.0)$ \\
\hline IHD before the reference AMI/ИБС до референсного ОИМ, n (\%) & $665(69.2)$ \\
\hline $\begin{array}{l}\text { Previous myocardial infarction/ } \\
\text { Ранее перенесенный инфаркт миокарда, n (\%) }\end{array}$ & $200(20.8)$ \\
\hline Heart failure/Сердечная недостаточность, n (\%) & $217(22.6)$ \\
\hline $\begin{array}{l}\text { Reference AMI with ST segment elevation/ } \\
\text { Референсный ОИМ с подъемом сегмента ST, n (\%) }\end{array}$ & $876(91.2)$ \\
\hline $\begin{array}{l}\text { Any complications during the reference AMI/ } \\
\text { Любые осложнения во время референсного ОИМ, n (\%) }\end{array}$ & $374(38.9)$ \\
\hline
\end{tabular}

ни. Хорошо видно, что частота назначения основных групп лекарственных препаратов, влияющих на прогноз жизни больных - антиагрегантов, ИАПФ, БАБ, статинов, оказалась достаточно высокой (это не касалось проведения тромболизиса, частота использования которого составила менее 10\%). Видно также, что частота применения многих групп препаратов в период ОИМ - тромболитиков, антиагрегантов, диуретиков, БАБ, статинов - существенно отличалась у выживших и умерших в отдаленном периоде больных.

При оценке ОР, скорректированного по возрасту и полу, обнаружилось значимое положительное влияние на выживаемость тромболизиса, назначения в стационаре БАБ и антиагрегантов (ОР 0,42, 0,65 и 0,58, соответственно).

На рис. 1 и 2 представлены кривые выживаемости Каплана-Мейера больных после ОИМ в зависимости от применения антиагрегантов до референсного ОИМ (рис. 1) и в стационаре во время референсного ОИМ (рис. 2). Очевидно, что если применение антиагрегантов до референсного ОИМ никак не повлияло на отдаленный прогноз жизни больных, то назначение этих же препаратов в стационаре существенно снижало риск смерти в отделенном периоде.

Table 2. Preparations received before the reference AMI and their influence on long-term life prognosis

Таблица 2. Препараты, принимавшиеся перед референсным ОИМ и их влияние на отдаленный прогноз жизни

\begin{tabular}{|c|c|c|c|c|c|c|}
\hline Drug group/Группа препаратов & Survived/Выжившие (n=770) & Deceased/Умершие $(n=191)$ & $p\left(\chi^{2}\right)$ & $\mathrm{RR} / \mathrm{OP}$ & СІ/ДИ & $p$ \\
\hline Diuretics/Диуретики & $14.0 \%$ & $10.5 \%$ & 0.196 & 0.67 & $0.41-1.10$ & 0.11 \\
\hline 及-blockers/ $\beta$-адреноблокаторы & $23.9 \%$ & $16.8 \%$ & 0.034 & 0.70 & $0.50-1.03$ & 0.07 \\
\hline ACE inhibitors/Ингибиторы АПФ & $36.9 \%$ & $30.4 \%$ & 0.092 & 0.66 & $0.47-0.93$ & 0.017 \\
\hline Antiplatelet agents/Антиагреганты & $16.2 \%$ & $13.6 \%$ & 0.373 & 0.76 & $0.48-1.19$ & 0.23 \\
\hline Statins/Статины & $2.2 \%$ & $1.1 \%$ & 0.302 & 0.68 & $0.17-2.72$ & 0.58 \\
\hline ССВ/Антагонисты кальция & $8.2 \%$ & $5.8 \%$ & 0.261 & 0.52 & $0.26-1.02$ & 0.058 \\
\hline
\end{tabular}

Table 3. Preparations received during hospital admission due to the reference AMI and their influence on long-term life prognosis

Таблица 3. Препараты, принимавшиеся во время стационарного периода по поводу референсного ОИМ и их влияние на отдаленный прогноз жизни

\begin{tabular}{|c|c|c|c|c|c|c|}
\hline Drug group/Группа препаратов & Survived/Выжившие (n=770) & Deceased/Умершие (n=191) & $p\left(\chi^{2}\right)$ & $\mathrm{RR} / \mathrm{OP}$ & СІ/ДИ & $p$ \\
\hline $\begin{array}{l}\text { Thrombolysis implementation/ } \\
\text { Проведение тромболизиса }\end{array}$ & $10.3 \%$ & $4.1 \%$ & 0.028 & 0.42 & $0.19-0,97$ & 0.043 \\
\hline Antiplatelet agents/Антиагреганты & $90.0 \%$ & $82.7 \%$ & 0.005 & 0.58 & $0.39-0,86$ & 0.0067 \\
\hline Anticoagulants/Антикоагулянты & $63.2 \%$ & $61.8 \%$ & 0.707 & 1.13 & $0.82-1,55$ & 0.46 \\
\hline Diuretics/Диуретики & $41.8 \%$ & $58.1 \%$ & 0.001 & 1.34 & $0.95-1,88$ & 0.092 \\
\hline$\beta$-blockers/ $\beta$-адреноблокаторы & $88.7 \%$ & $79.6 \%$ & 0.001 & 0.65 & $0.44-0,96$ & 0.030 \\
\hline ACE inhibitors/Ингибиторы АПФ & $89.5 \%$ & $92.0 \%$ & 0.656 & 0.96 & $0.59-1,55$ & 0.86 \\
\hline Statins/Статины & $54.7 \%$ & $64.9 \%$ & 0.011 & 0.88 & $0.64-1,23$ & 0.46 \\
\hline ССВ/Антагонисты кальция & $4.6 \%$ & $7.9 \%$ & 0.065 & 1.42 & $0.80-2,52$ & 0.23 \\
\hline
\end{tabular}




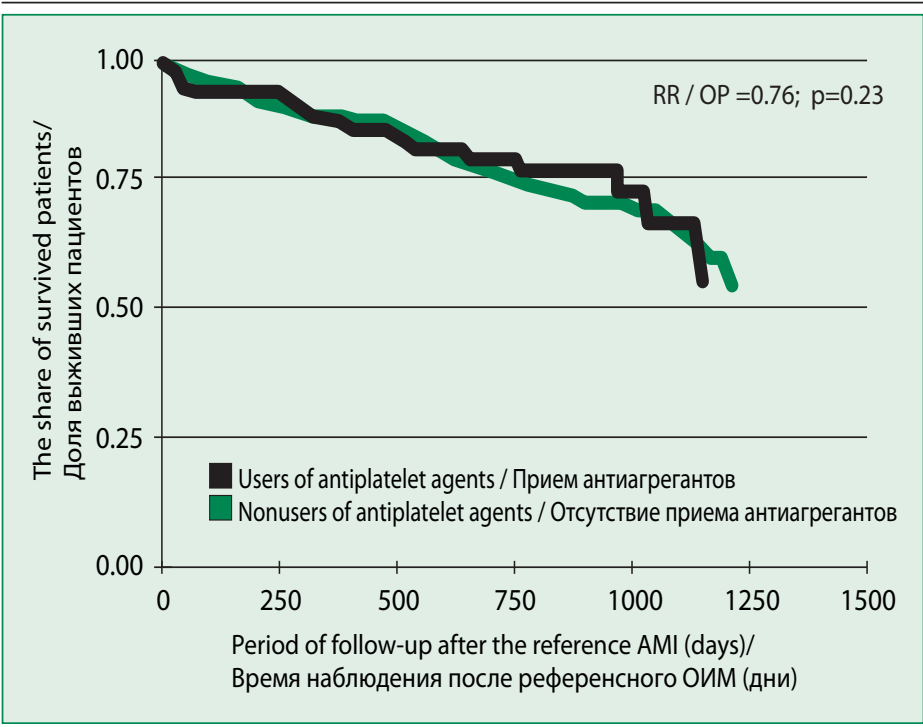

Figure 1. Influence of antiplatelet agents prescribed before the reference AMI on long-term life prognosis $(n=961)$

Рисунок 1. Влияние антиагрегантов, назначенных до референсного ОИМ, на отделенный прогноз жизни (n=961)

rather high, this did not concern thrombolytics, which were provided in less than $10 \%$ of the cases. One can also see that rate of intake of many preparations (thrombolytics, antiplatelet agents, diuretics, $\beta$-blockers, statins) during AMI significantly varied in survived patients and deceased ones in late terms.

At RR evaluation after adjustment for age and gender, thrombolytics, $\beta$-blockers and antiplatelet agents prescribed in hospital revealed significant positive influence on survival rate (RR $0.42,0.65$ and 0.58 , respectively).

Kaplan-Meier curves of survival after AMI depending on antiplatelet agents use before and during the reference $\mathrm{AMI}$ are presented in Figures 1 and 2 , respectively. It is evident that while antiplatelet agents prescribed before the reference AMI did not influence long-term life prognosis, the same drugs administrated in hospital significantly reduced death risk in late terms.

Figure 3 presents data about treatment of 632 survived patients, received at their repeated visit. Rate of the main drug groups prescription (ACE inhibitors, antiplatelet agents, $\beta$-blockers and statins) was rather high. It is necessary to mention that these data were received from out-patient medical records; special questionnaires evaluating prescribed treatment adherence were not used in the "LIS" study.

\section{Discussion}

This part of the "LIS" study was aimed at evaluation of drug treatment in patients survived the acute stage of the disease and discharged from hospital. We have succeeded in estimation of the drugs use
На рис. 3 представлены данные о назначенной лекарственной терапии, полученные при повторном визите выживших 632 больных. Частота назначения основных групп лекарственных препаратов (ИАПФ, антиагрегантов, БАБ, статинов) была достаточно высокой. Следует оговориться, что, как отмечалось выше, эти данные вносились в карту обследования лечащими врачами из амбулаторных карт; специальные анкеты, отслеживающие приверженность назначенной терапии, в исследовании ЛИС не использовались.

\section{Обсуждение}

В данной части исследования ЛИС отслеживалась лекарственная терапия больных, выживших после острой стадии болезни и выписанных из стационара. Нам удалось отследить частоту использования лекарственных препаратов в различные периоды болезни: перед развившимся референсным ОИМ, непосредственно во время референсного ОИМ, т.е. в стационаре, а также через определенный срок после референсного ОИМ (в среднем через 1,7士0,9 лет). В первых двух случаях информация оценивалась по историям болезни, в последнем случае - врачами при визитах больных в поликлинику, причем это касалось только выживших больных и больных, согласившихся придти на повторный визит (их оказалось 632 человека).

Полученные данные, во-первых, позволили оценить реальную частоту использования конкретных препаратов на разных этапах болезни, во-вторых, они позволили в определенной степени (с рядом оговорок) оценить прогности-

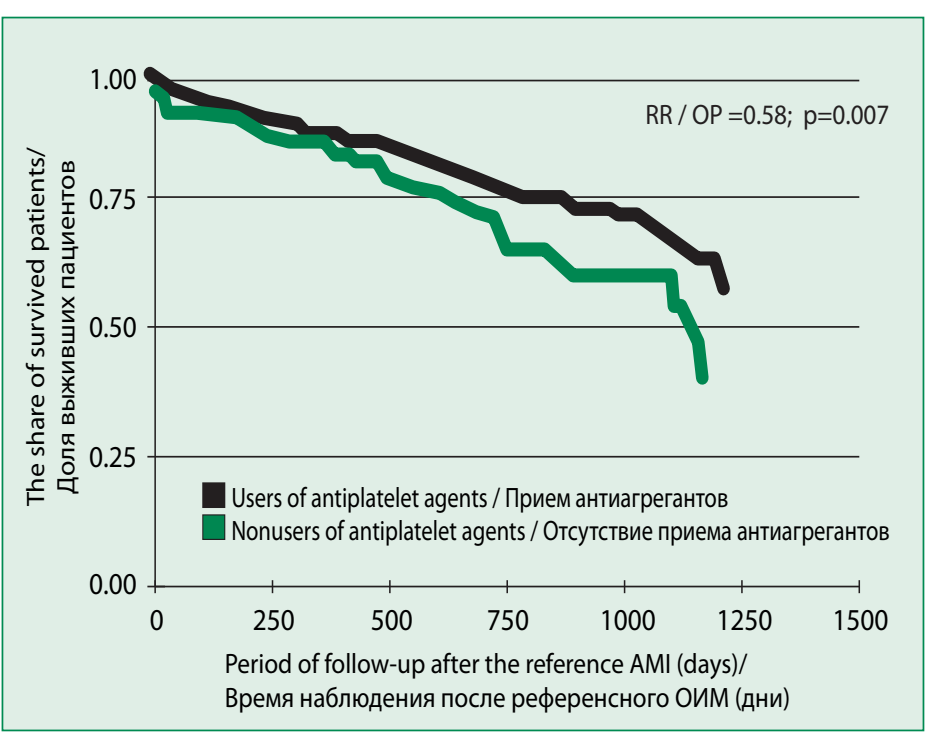

Figure 2. Influence of antiplatelet agents prescribed during the reference $A M I$ in hospital on long-term life prognosis $(n=961)$

Рисунок 2. Влияние антиагрегантов, назначенных в стационаре во время референсного ОИМ, на отделенный прогноз жизни $(n=961)$ 


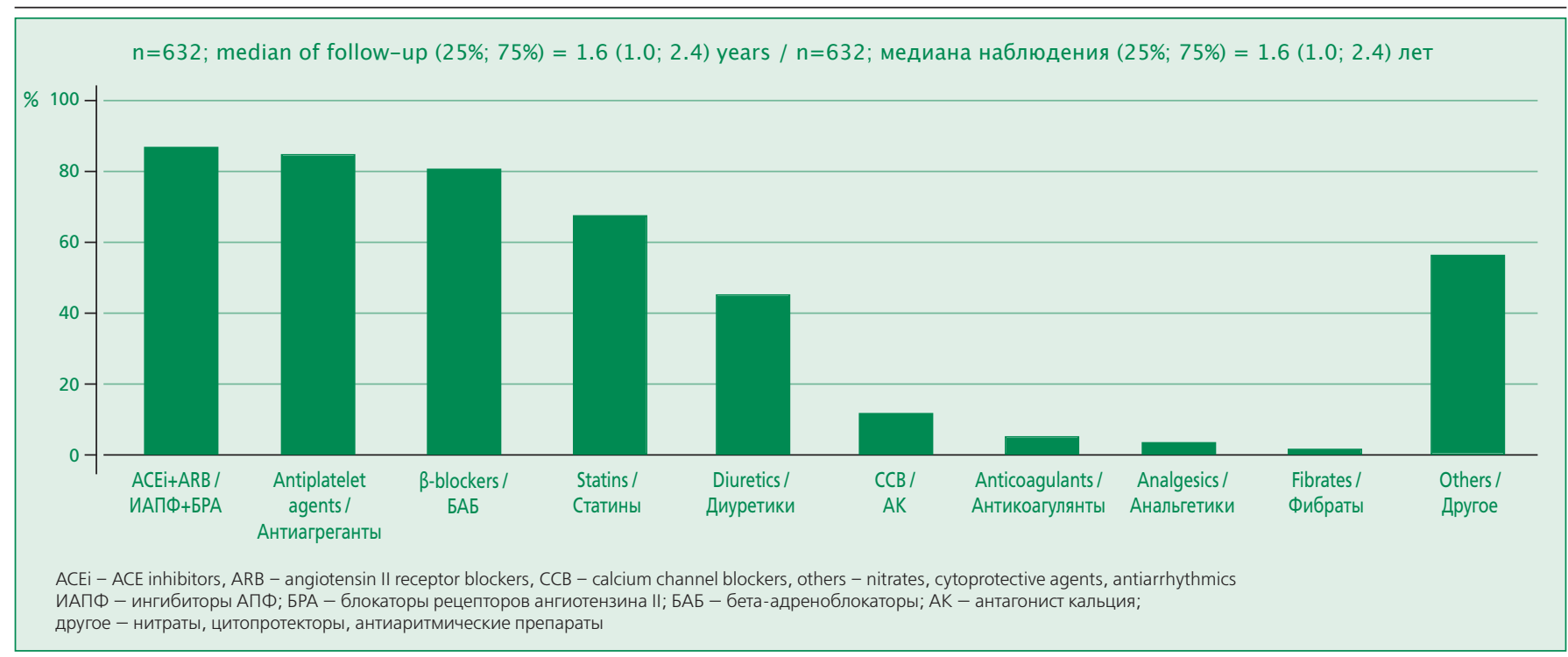

Figure 3. Data about drugs prescription in distant terms after the reference AMI

Рисунок 3. Данные о назначении лекарственных препаратов больным в отдаленные сроки после референсного ОИМ

frequency in different periods of the disease: before the reference $\mathrm{AMI}$, during it (in hospital) and some time later (on the average after $1.7 \pm 0.9$ years). In the first two cases information was received from patient's charts, in the last one - at patient's visits to out-patient clinic, at that this only concerned to survived patients who consented to come for the repeated examination (632 persons).

Firstly, received data allowed to evaluate the real incidence of specific drugs use at different stages of the disease, secondly, these data to some extent allowed to estimate prognostic value of certain drug groups. For example, we have managed to determine prognostic value of treatment prescribed before and during the reference AMI.

Certainly, diseases registers do not implicate any interference in therapy and so cannot compete with CCT in terms of assessment of a drug influence on disease outcomes [21]. However, it is well known that such registers allow to evaluate a drug effect, foremost in cases when some patients do and some do not intake a drug, in other words - when two groups of patients are naturally formed and distinct from each other by the fact of intake of a certain medication (users and nonusers) [16]. Unlike CCT these groups of patients can also differ from each other in some other parameters (such as age, gender, disease severity and so on), however, statistic methods let adjust these indices. Thus, in the framework of the "LIS" study we evaluated prognostic value of medications with the adjustment for age and gender of the patients.

According to the "LIS" study results some preparations, received before the reference AMI, demonstrated favorable effect on long-term life prognosis. ческое влияние конкретных групп лекарственных препаратов. Так, удалось оценить влияние лечения, назначаемого перед референсным ОИМ и во время него, на прогноз жизни больного.

Безусловно, регистры заболеваний не подразумевают какого-либо вмешательства в терапию больного и поэтому ни в коем случае не могут конкурировать с ККИ в плане оценки влияния препарата на исходы болезни [21]. Тем не менее, хорошо известно, что в рамках регистров вполне реально попытаться оценить эффект лекарственного препарата, в первую очередь в тех случаях, когда часть больных принимает, а часть не принимает препарат. Т.е., естественно формируются две группы больных, отличающихся между собой по факту применения (или неприменения) конкретного препарата [16]. В отличие от ККИ, эти группы принимавших и не принимавших препарат пациентов могут различаться между собой и по ряду других признаков (возрасту, полу, тяжести заболевания и пр.), тем не менее, статистические методы позволяют ввести поправки на эти признаки. Так, в исследовании ЛИС прогностический эффект лекарственных препаратов оценивался только с поправкой на возраст и пол пациентов.

Оценивая полученный в исследовании ЛИС вклад в показатели смертности конкретных лекарств, отметим в первую очередь тот факт, что ряд препаратов, принимавшихся до референсного ОИМ, благоприятно повлияли на отдаленный прогноз жизни, т.е. на то, что произошло после перенесенного ОИМ. Это касалось двух классов препаратов: БАБ и ИАПФ. Результат представляется вполне логичным, учитывая результаты ККИ, проводившиеся с данными препаратами $[3,22,23]$. Отсутствие эффекта от применения статинов и антиагрегантов, которые теоретически должны были бы также проявить себя в данной ситуации, возможно, объясняется незначительным количеством больных, их принимавших. 
It refers to $\beta$-blockers and ACE inhibitors. The results are in line with data of the CCT carried out with these drugs $[3,22,23]$. Lack of beneficial effect in case of statins and antiplatelet agents, which theoretically should have approved themselves in such a situation, can possibly be explained by insufficient amount of patients received these drugs.

Conclusion of this part of the "LIS" study is very important for practice: secondary drug prevention in patients at high risk of AMI can not only benefit life prognosis in acute stage of the disease (see the first part of the article), but also improve long-term survival rate. Such a low incidence of the main drug groups prescription for primary and secondary prevention, demonstrated in the "LIS" study, probably impact on high mortality rate, revealed at long-term follow-up. It is to be recalled that the main part of the study showed that about half of the patients ( 40\%) admitted to hospital due to $\mathrm{AMI}$, die during the first three years from the start of the disease [19].

Data about influence of the drugs prescribed in acute stage of the disease on long-term prognosis were not less important. Obvious beneficial effect was demonstrated by thrombolytics, antiplatelet agents and $\beta$-blockers. What is in line with the CCT data [1, $2,6]$. We did not reveal influence of statins on the patients' survival rate which can be explained by different reasons. Perhaps, these medications were taken in insufficient doses and target level of low-density lipoprotein cholesterol could not be achieved. Besides, adherence to the treatment with these drugs might have been rather low.

Evaluation of drug therapy at the repeated visit (in those who survived and agreed to come to the repeated examination), on the one hand, revealed rather favorable picture: prevalence of the main drug groups prescription, used as secondary prevention after reference AMI, including statins, was fairly high (Fig.3). On the other hand, these data only take into account survived patients (we do not know, how the deceased were treated), at that among survived we only examined those who agreed to come to an appointment. We do not also know how the patients took the prescribed drugs as the "LIS" study was not aimed at evaluation of therapy adherence. However these data testify that doctors generally competently administrate drug treatment to such patients.

\section{Conclusion}

So, evaluation of drug treatment in AMI patients at different stages of the disease (including one before AMI advent), carried out in the framework of the "LIS" study, revealed very low prevalence of mod-
Вывод этой части исследования ЛИС представляется крайне важным для практики: вторичная лекарственная профилактика у больных с высоким риском ОИМ может не только улучшить прогноз жизни в острой стадии болезни (об этом писалось в первой части статьи) , но и положительно повлиять на отдаленные показатели выживаемости больных. Возможно, столь низкая частота назначения основных групп лекарственных препаратов, используемых для первичной и вторичной профилактики, продемонстрированная в исследовании ЛИС, во многом объясняет те высокие показатели смертности, выявленные при длительном наблюдении больных. Напомним, что основная часть исследования показала, что почти около половины больных ( 40\%), поступающих с диагнозом ОИМ, умирает в течение первых трех лет с момента начала болезни [19].

Не менее важными оказались данные о влиянии препаратов, назначенных в острой стадии болезни, на отделенный прогноз жизни больных. В этом случае отчетливо проявили себя тромболизис, антиагреганты, а также БАБ, что в принципе также четко совпадает с данными, полученными в КKИ $[1,2,6]$. Отсутствие явного эффекта статинов на выживаемость больных можно объяснять различными причинами. Возможно, эти препараты принимались в недостаточных дозах, вследствие чего не достигались так называемые целевые цифры холестерина липопротеидов низкой плотности, возможно также, что приверженность терапии этими препаратами была очень невысокой.

Изучение назначаемой лекарственной терапии, проведенное при повторном визите больных (только у тех, кто выжил и согласился придти на повторный прием), показало, с одной стороны, довольно благоприятную картину: частота назначения основных групп лекарственных препаратов, использующихся для вторичной профилактики после референсного ОИМ, в том числе статинов, оказалась достаточно высокой (рис. 3). С другой стороны, следует учитывать, что эти данные имеются только для выживших больных (неизвестно, как лечились умершие больные), а среди выживших - только для тех, кто согласился придти на повторный прием. Неизвестно также, как реально принимали эти пациенты назначенные им препараты, так как приверженность терапии в исследовании ЛИС не оценивалась, о чем уже говорилось выше. Тем не менее, данные в принципе свидетельствуют о том, что врачи в целом грамотно подходят к назначению лекарственной терапии таким пациентам.

\section{Заключение}

Таким образом, изучение лекарственной терапии у больных ОИМ на разных стадиях болезни (в том числе и перед ее развитием), проведенное в рамках исследования ЛИС, выявило крайне невысокий охват современной лекарственной терапией больных перед развившемся у них ОИМ, несмотря на то, что вероятность ОИМ была достаточно очевидна. Не менее важно и то, что данное исследование до- 
ern drug therapy in high-risk patients before AMI advent. Not less important was the fact proved by the "LIS" study that if the treatment before the disease occurrence was more active, long-term survival prognosis would have been much better even despite occurred AMI. It is quite likely that high long-term mortality rate registered in the "LIS" study is to a considerable degree explained by inadequate treatment in patients at high cardiovascular risk. This one more time underlines the importance of drug prevention (both primary and secondary) in persons at high risk of cardiovascular complications.

Disclosures. All authors have not disclosed potential conflicts of interest regarding the content of this paper. казало, что будь терапия до развития болезни более активной, даже несмотря на произошедший ОИМ, отдаленный результат в плане выживаемости был бы значительно лучше. Весьма вероятно, что крайне высокие показатели отдаленной смертности больных, зарегистрированные в исследовании ЛИС, в значительной степени объясняются неадекватной терапией пациентов с высоким риском сердечно-сосудистых осложнений в целом, что еще раз подчеркивает важнейшую роль лекарственной профилактики (как первичной, так и вторичной) у лиц с высоким риском сердечно-сосудистых осложнений.

Конфликт интересов. Все авторы заявляют об отсутствии потенциального конфликта интересов, требующего раскрытия в данной статье.

\section{References / Литература}

1. Baigent C, Collins R, Appleby P, et al. ISIS-2: 10 year survival among patients with suspected acute myocardial infarction in randomised comparison of intravenous streptokinase, oral aspirin, both, or neither. The ISIS-2 (Second International Study of Infarct Survival) Collaborative Group. BMJ 1998:316(7141):1337-1343.

2. A randomized trial of propranolol in patients with acute myocardial infarction. I. Mortality results. JAMA 1982;247(12):1707-1714.

3. Lonn E, Shaikholeslami R, Yi Q, et al. Effects of ramipril on left ventricular mass and function in cardiovascular patients with controlled blood pressure and with preserved left ventricular ejection fraction: a substudy of the Heart Outcomes Prevention Evaluation (HOPE) Trial. J Am Coll Cardiol 2004:43(12):2200-2206.

4. Kober L, Torp-Pedersen C, Carlsen JE, et al. A clinical trial of the angiotensin-converting-enzyme inhibitor trandolapril in patients with left ventricular dysfunction after myocardial infarction. Trandolapril Cardiac Evaluation (TRACE) Study Group. N Engl J Med 1995;333(25):1670-1676.

5. Schwartz GG, Olsson AG, Ezekowitz MD, et al. Effects of atorvastatin on early recurrent ischemic events in acute coronary syndromes: the MIRACL study: a randomized controlled trial. JAMA 2001:285(13):1711-1718.

6. Olsson G, Wikstrand J, Warnold I, et al. Metoprolol-induced reduction in postinfarction mortality: pooled results from five double-blind randomized trials. Eur Heart J 1992;13(1):28-32.

7. Ford ES, Ajani UA, Croft JB, et al. Explaining the decrease in U.S. deaths from coronary disease, 19802000. N Engl J Med 2007;356(23):2388-2398

8. Boersma E, Keil U, De Bacquer D, et al. Blood pressure is insufficiently controlled in European patients with established coronary heart disease. J Hypertens 2003;21(10):1831-1840.

9. Kobalava Zh.D., Kotovskaya Yu.V., Sklizkova L.A., Moiseev V.S. Treatment and screening of elderly patients with hypertension: Perceptions of physicians and actual practice (according to the Russian scientific-practical program ARGUS) Arterial'naya Gipertenziya 2002;8(5):165-168. Russian (Кобалава Ж.Д., Котовская Ю.В., Склизкова Л.А., Моисеев В.С. Лечение и обследование пожилых больных с артериальной гипертонией: представления врачей и реальная практика (по данным Российской научно-практической программы АРГУС). Артериальная Гипертензия 2002;8(5):165-168).

10. Yusuf S, Islam S, Chow CK, et al. Use of secondary prevention drugs for cardiovascular disease in the community in high-income, middle-income, and low-income countries (the PURE Study): a prospective epidemiological survey. Lancet 2011;378(9798):1231-1243.

11. Kotseva K, Wood D, De Backer G, De Bacquer D, Pyorala K, Keil U. EUROASPIRE III: a survey on the lifestyle, risk factors and use of cardioprotective drug therapies in coronary patients from 22 European countries. Eur J Cardiovasc Prev Rehabil 2009;16(2):121-137.

12. Larsen J, Andersen M, Bjerrum L, et al. Insufficient use of lipid-lowering drugs and measurement of serum cholesterol among patients with a history of myocardial infarction. J Cardiovasc Risk 2003;10(1):61-64
13. Alpert JS. Are data from clinical registries of any value? Eur Heart J 2000;21 (17):1399-1401

14. Dyke CK, Calif RM. National and regional registries: what good are they? Eur Heart J 2000;21(17):14011403.

15. Fox KA. Registries and surveys in acute coronary syndrome. Eur Heart J 2006;27(19):2260-2262

16. Krumholz HM, Chen J, Chen YT, et al. Predicting one-year mortality among elderly survivors of hospitalization for an acute myocardial infarction: results from the Cooperative Cardiovascular Project. J Am Coll Cardiol 2001;38(2):453-459.

17. Horinaka S, Yabe A, Yagi H, et al. Effects of nicorandil on cardiovascular events in patients with coronary artery disease in the Japanese Coronary Artery Disease (JCAD) study. Circ J 2010;74(3):503509

18. Martsevich S.Yu., Ginzburg M.L., Kutishenko N.P., et al. The LIS study (Lubertsy mortality study of patients with acute myocardial infarction): a portrait of the patient. Kardiovaskularnaya Terapiya i Profilaktika 2011;10(6):89-93.Марцевич С.Ю., Гинзбург М.Л., Кутишенко Н.П., и соавт. Исследование ЛИС (Люберецкое исследование смертности больных, перенесших острый инфаркт миокарда): портрет заболевшего. Кардиоваскуларная Терапия и Профилактика 2011;10(6):89-93.

19. Martsevich S.Yu., Ginzburg M.L., Kutishenko N.P., et al. Lubertsy study on mortality in patients with acute myocardial infarction. The first results of the study «LIS》. Klinitsist 2011;2:24-27. Russian (Mapцевич С.Ю., Гинзбург М.Л., Кутишенко Н.П., и соавт. Люберецкое исследование по изучению смертности больных, перенесших острый инфаркт миокарда. Первые результаты исследования «ЛИС». Клиницист 2011;2:24-27).

20. Martsevich S.Yu., Ginzburg M.L., Kutishenko N.P., et al. Lubertsy mortality study of patients with acute myocardial infarction (LIS): analysis of anamnestic factors determining death in the hospital. Kardiovaskularnaya Terapiya i Profilaktika 2012; 11 (1), 45-48. Russian (Марцевич С.Ю., Гинзбург М.Л., Кутишенко Н.П., и др. Люберецкое исследование смертности больных, перенесших острый инфаркт миокарда (ЛИС): анализ анамнестических факторов, определяющих смерть в стационаре. Кардиоваскуларная Терапия и Профилактика 2012; 11(1): 45-48).

21. MacMahon S, Collins R. Reliable assessment of the effects of treatment on mortality and major morbidity, II: observational studies. Lancet 2001;357(9254):455-462.

22. Tuomilehto J, Wikstrand J, Olsson G, et al. Decreased coronary heart disease in hypertensive smok ers. Mortality results from the MAPHY study. Hypertension 1989;13(6 Pt 2):773-780.

23. Fox KM, Henderson JR, Bertrand ME, et al. The European trial on reduction of cardiac events with perindopril in stable coronary artery disease (EUROPA). Eur Heart J 1998;19 Suppl J:J52-55.

Received/Поступила 17.07.2012 Accepted/Принята в печать 24.07.2012 